\title{
Tranexamic Acid Increases Early Postoperative Pain and Decreases Time to First Opioid Following Total Knee Arthroplasty
}

\author{
Sachin Seetharam ${ }^{1}$, Sydney Keller ${ }^{1}$, \\ Mary Ziemba-Davis², R. Michael Meneghini MD²,3 \\ ${ }^{1}$ Indiana University School of Medicine \\ 2 Indiana University Health Physicians, Orthopaedics \\ ${ }^{3}$ Indiana University School of Medicine, Department of Orthopaedic Surgery \\ Background and Hypothesis: Tranexamic acid (TXA) decreases blood loss in \\ total knee arthroplasty (TKA). However, TXA evoked pain in rats by inhibiting \\ GABA and glycine receptors in the spinal dorsal horn, and caused cellular death \\ in ex vivo and in vitro human periarticular tissues exposed to clinical \\ concentrations of TXA. We evaluated inpatient postoperative pain and blood loss \\ in TKA performed with and without TXA. \\ Project Methods: 105 consecutive cemented TKAs without TXA were compared \\ to 72 consecutive cemented TKAs with TXA. Procedures were performed by a \\ single surgeon using identical perioperative medical and pain-control protocols. \\ Outcomes included: average of q2-4 hour pain scores during the first 24 hours \\ after PACU discharge, average pain during remainder of stay, final pain score \\ prior to discharge, time in minutes to first opioid after PACU discharge, total \\ opioids in morphine equivalents (MEQs) during the first 24 hours after PACU \\ discharge, average MEQs per remaining days of stay, and mean g/dL pre- to \\ postoperative decrease in hemoglobin. Multivariate analyses accounted for 15 \\ demographics and covariates.
}

Results: The sex $(p=0.393)$, age $(p=0.784)$, and BMI $(p=0.930)$ of the two cohorts were similar. Mean pain during the first 24 hours was greater (4.1 vs. 3.2, $p=0.001)$, MEQs consumed during the first 24 hours were greater (45 vs. 37 , $p=0.069$ ), and time to first opioid medication was shorter (326 vs. $414, p=0.023$ ) in patients who received TXA. The decrease in hemoglobin was less in patients who received TXA (-2.2 vs. $-2.7, \mathrm{p}<0.001)$.

Conclusion and Potential Impact: Our hypothesis based on animal and laboratory studies that TXA may increase early postoperative pain was confirmed by three metrics. Consistent with the effective life of TXA, pain and opioid consumption after 24 hours did not differ based on TXA use. Further work is warranted to investigate the nature consequences associated with TXA, relative to its demonstrated benefits for blood conservation. 